\title{
Análisis comparativo de dos selladores radiculares a base de ZOE y resina epóxica
}

\author{
Comparative analysis of two root sealers based on ZOE and epoxy resin
}

Eunice Lazcano-Dorantes ${ }^{a}, J_{\text {. Eliezer Zamarripa-Calderón }}{ }^{b}$, Adriana L. Ancona-Meza ${ }^{c}$,

Ana J. Monjarás-Ávila ${ }^{d}$, Carlos E. Cuevas-Suárez ${ }^{e}, J^{\text {Alejandro Rivera-Gonzaga }}{ }^{f}$

\begin{abstract}
:
The use of endodontic posts is indicated to help the retention of the final restoration, they are usually made of fiberglass and allow the tooth to recover its functionality. Before its placement, it is necessary to perform a three-dimensional sealing of the root canals, for this purpose, gutta-percha filling material and root canal sealers are used. There is evidence that the presence of eugenol in sealers affects the polymerization of resin cements, which in turns decrease the adhesion of the post. For this reason, the aim of this work was to compare the push-out strength, and additionally, the microfiltration and radiographic density of two root sealers in bovine teeth roots. Two commercially available endodontic root canal sealers were used: VIARDEN® (zinc oxide and eugenolbased sealer) and ADSEAL ${ }^{\mathrm{TM}}$ (epoxy resin-based sealer). Data was analyzed using a significance level of $\alpha=0.05$. The push-out bond strength test showed that the group sealed with ADSEAL ${ }^{\mathrm{TM}}$ sealer obtained the highest values; the microfiltration results showed that ZOE-based sealer had the highest values $(p<0.05)$ and the radiographic density was statistically similar $(p>0.05)$. From de analysis performed, it is concluded that the chemical composition of the material used as an endodontic sealer has a significant influence on the apical microfiltration and push-out bond strength. The epoxy resin-based sealer seems to have the best performance so it can be considered as ideal for endodontic procedures.
\end{abstract}

\section{Keywords:}

Root sealer, adhesion, eugenol, resin, endodontics

\section{Resumen:}

El objetivo clínico de un endoposte es ayudar en la retención de la restauración final, generalmente son de fibra de vidrio y permiten que el diente recobre su funcionalidad. Antes de este procedimiento clínico, es necesario realizar un sellado tridimensional de los conductos radiculares, utilizando gutapercha y un sellador radicular. Hay evidencia de que la presencia de eugenol en los selladores afecta la polimerización de cementos de resina lo que disminuye la adhesión del poste, por esta razón el objetivo de este trabajo fue comparar la resistencia adhesiva, la microfiltración y la densidad radiográfica de dos selladores radiculares en raíces de dientes bovinos. Dos selladores endodónticos disponibles comercialmente fueron utilizados: VIARDEN® (sellador a base de óxido de zinc y eugenol) y ADSEAL ${ }^{\mathrm{TM}}$ (sellador a base de resina epóxica). Todos los datos fueron analizados utilizando un nivel de significancia de $\alpha=0.05$. La prueba de resistencia adhesiva al desalojo demostró que el grupo obturado con el sellador ADSEAL ${ }^{\mathrm{TM}}$ obtuvo los valores más elevados; los resultados de microfiltración mostraron que el sellador a base de ZOE tuvo los mayores valores ( $<<0.05$ ) y la densidad radiográfica observada fue estadísticamente similar ( $>>0.05$ ). Del análisis efectuado, se concluye que la composición química del material utilizado como sellador endodóntico tiene una influencia significativa en la microfiltración apical y en la resistencia adhesiva. Por otro lado, el sellador a base de resina epóxica parece tener el mejor desempeño por lo que puede considerarse como ideal para los procedimientos endodónticos.

\section{Palabras Clave:}

Sellador radicular, adhesión, eugenol, resina, endodoncia

\footnotetext{
${ }^{a}$ Universidad Autónoma del Estado de Hidalgo, Instituto de Ciencias de la Salud, Maestría en Ciencias Biomédicas y de la Salud, Email: sshsegundonivelsaludbucal@gmail.com

${ }^{\text {b}}$ Universidad Autónoma del Estado de Hidalgo, Instituto de Ciencias de la Salud, ORCID: 0000-0001-5830-5550, Email: eliezerz@uaeh.edu.mx c Universidad Autónoma del Estado de Hidalgo, Instituto de Ciencias de la Salud, Email: ancona@uaeh.edu.mx 


\section{Introducción}

La OMS ha definido la caries dental como un proceso localizado de origen multifactorial que se inicia después de la erupción dentaria, determinando el reblandecimiento del tejido duro del diente y que evoluciona hasta la formación de una cavidad: ${ }^{1}$ es un proceso multifactorial cuya infección y actividad se establecen mucho antes de que ocurra la cavitación mediado por la presencia de una biopelícula que puede alojar bacterias cariogénicas que sintetizan glucanos a partir de los carbohidratos de la placa dentobacteriana cuyo metabolismo produce ácidos, los cuales disminuyen el $\mathrm{pH}$ de la biopelícula y afecta el esmalte, causando la pérdida de mineral en la estructura dental. ${ }^{2}-{ }^{4}$ Existen diversos tratamientos para la caries dental que dependen del área afectada, la profundidad y otras características propias en cada paciente. Los principales son remineralización, obturaciones estéticas o metálicas, directas o indirectas y endodoncia.

El tratamiento endodóntico se realiza en órganos dentarios debido a caries, traumatismos o indicación protésica; se basa al principio en la tríada de desbridamiento, desinfección exhaustiva y obturación, todos con la misma importancia. La gutapercha es el material más usado para la obturación; sus principales ventajas son: plasticidad, fácil manipulación, mínima toxicidad, radiopacidad y fácil eliminación con calor o disolventes. Las desventajas incluyen falta de adhesión a la dentina y retracción al enfriarse. ${ }^{5}$

La función principal de un sellador radicular es llenar los espacios entre el material del núcleo y las paredes del canal de la raíz y entre los conos de gutapercha, en un intento de formar una masa coherente de material de obturación sin espacios vacíos. Se espera que el sellador pueda rellenar irregularidades y discrepancias menores entre la obturación y las paredes del conducto, y de la misma manera, los conductos accesorios y túbulos dentinarios. Los selladores a base de óxido de zinc y eugenol tienen acción germicida por lo que también se utilizan para destruir las bacterias que quedan después de la limpieza y conformación del conducto radicular. ${ }^{5},{ }^{6}$ Los cementos selladores de óxido de zinc-eugenol experimentan reabsorción si pasan a los tejidos perirradiculares, presentan largo tiempo de fraguado, se contraen al fraguar, se pueden disolver y teñir la estructura dental, sin embargo, poseen actividad antimicrobiana. El primer sellador radicular utilizado fue introducido por Rickert y Dixon; contenía partículas de plata para aportar radiopacidad, pero tenía el inconveniente de teñir la estructura dental. En 1958 Grossman modificó la composición e introdujo una formulación que no producía tinción: el cemento sellador de Roth. En la actualidad existen diferentes selladores a base de ZOE con diferente tiempo de fraguado, presentación, consistencia e ingredientes adicionales. ${ }^{5}$ Los cementos selladores de resina proporcionan adhesión y no contienen eugenol. ADSEAL ${ }^{\mathrm{TM}}$ de la casa comercial MetaBiomed es un sellador que tiene propiedades fisicoquímicas aceptables, biocompatibilidad y capacidad de sellado similares al sellador AHplus, que es una resina epoxi de fraguado lento que no libera formaldehído al fraguar. $^{7}$

Se han realizado numerosos estudios en torno al uso de selladores a base de ZOE: en el año 2015, Adiel Skupien y col determinaron que la presencia de eugenol afecta la polimerización de cementos de resina y, por tanto, a la adhesión del poste. ${ }^{8}$ En el mismo año, Collares y col. realizaron un metaanálisis sobre la influencia del eugenol en la fuerza adhesiva de postes de fibra cementados en conductos radiculares, y concluyeron que el sellador a base de eugenol reduce la adhesión de postes de fibra cementados en raíces dentales con cemento de resina, sin importar el tipo de sistema adhesivo o cemento de resina utilizada. ${ }^{9}$ Mosharraf y Zare en 2014, obtuvieron un resultado similar cuando compararon los selladores AHplus y Endofill: encontraron diferencias significativas que sugieren que el eugenol (Endofill) redujo significativamente la resistencia de la unión de los postes de fibra cementadas con cemento de resina. ${ }^{10}$

Demiryürek y col. en 2010, evaluaron los efectos de tres selladores endodónticos en la adhesión de un poste de fibra unido con cemento de resina adhesiva. Utilizaron los selladores AHplus Endofill y Sealapex y encontraron que el tipo de sellador de canal y la preparación químicomecánica de los conductos radiculares afecta a la adhesión de postes de fibra cementados con cemento de resina. $^{11}$

Menezes y col. en el 2008, comprobaron que la composición del cemento endodóntico y el tiempo transcurrido entre el llenado de la raíz y la colocación del poste de fibra de vidrio interfiere con la adhesión a la dentina del conducto radicular; utilizando la prueba de push out y sellador a base de $\mathrm{Ca}(\mathrm{OH})_{2}$, a base de resina (AH 26) y a base de ZOE (Endofill), encontraron que el sellador Endofill interfirió negativamente con la adhesión cuando la cementación del poste se retrasó siete días. ${ }^{12}$ Sin embargo, en el año 2010, Souza y col. evaluaron la influencia del eugenol de selladores endodónticos y la estrategia de cementado en la adhesión de postes de fibra de vidrio con incisivos bovinos, obturando solo con gutapercha o con sellador a base de ZOE, y concluyeron que el sellador con eugenol no influyó en los valores de resistencia adhesiva del endoposte cementado con resina con los diferentes sistemas de cementación. ${ }^{13}$

Por otro lado, Souza-Neto y col. evaluaron la influencia de un sellador endodóntico con eugenol (EndoFill) sobre la adhesión de los postes intra-radiculares cementados 
con un cemento a base de resina y un cemento de fosfato de zinc y concluyeron que el cemento a base de fosfato de zinc mostró una mayor retención del poste que el cemento a base de resina lo que demostró que el sellador que contenía eugenol interfirió con las propiedades adhesivas de cemento a base de resina. ${ }^{14}$ Días y col. en el 2009, evaluaron la influencia de los selladores a base de eugenol sobre la adhesión en los postes de acero inoxidable cementados con fosfato de zinc y Panavia ${ }^{\mathrm{TM}}$ en diferentes momentos después de la obturación del conducto radicular, en el cual fue utilizado un sellador a base de resina y utilizando la prueba pullout. Mostraron que el sellador a base de eugenol influye en la resistencia a la tracción de los postes colocados con cemento de resina, sin embargo, no existe influencia en el tiempo esperado entre la obturación del conducto radicular, la preparación y el cementado del poste. ${ }^{15}$

Izadi y col. en el año 2013, evaluaron la influencia del sellador con eugenol en la adhesión de endopostes radiculares utilizando un cemento a base de resina, con el sellador Endofill y el cemento Panavia ${ }^{\mathrm{TM}}$ y postes del número I, II y III, llegaron a la conclusión que el sellador con eugenol reduce la retención de postes cementados con cemento de resina y que si se elimina mayor dentina se aumenta la retención del poste de fibra de vidrio. ${ }^{16}$

De manera similar, en el año 2012, Khalil Aleisa y col. evaluaron la resistencia adhesiva, con tres tipos de selladores, dos con eugenol, EndoFill, Tubli-Seal, y uno a base de resina $\mathrm{AH} 26$, con tres diferentes tipos de cementos de resina compuesta; Rely $X$ Unicem, ParaCore y Variolink II. Comprobaron que los selladores a base de eugenol demuestran un descenso significativo en la fuerza de adhesión para los postes de fibra cementados con cemento de resina; Rely $X$ Unicem mostró mayor resistencia a la adhesión que ParaCore y Variolink II cuando se utilizó un sellador a base de eugenol. $^{17}$

Da Silva en el 2012 evaluó la influencia del eugenol en la adhesión del endoposte metálico prefabricado con cemento de resina, para esto utilizó los selladores $\mathrm{AH}$ Plus, Sealapex y Endofill; los postes se cementaron con RelyX ARC y, nuevamente, verificó que el eugenol influye en la resistencia de la unión de los postes cuando se utiliza cemento resinoso. ${ }^{18}$

Mosharraf y Haerian en el año 2011, evaluaron mediante la técnica push-out la adhesión de postes de fibra de vidrio cementados con dos diferentes resinas, sistema de autograbado (Panavia F2.0 / ED PRIMER II) y sistema convencional (Variolink II / Excite DSC). Al realizarse el análisis estadístico no hubo diferencia significativa entre la media de resistencia adhesiva de los sistemas de cementos de resina convencionales y los de autograbado, sin embargo, la región coronal de la dentina radicular mostró una resistencia de unión significativamente más alta que la región apical. ${ }^{19}$

Özcan y col. en el 2012, evaluaron el efecto de un sellador a base de eugenol y cómo afecta a la adhesión del endoposte cuando se utilizan 3 cementos a base de resina: un sistema adhesivo de autograbado de 2 pasos, Clearfil Liner Bond 2V Panavia ${ }^{\mathrm{TM}} \mathrm{F}$, un sistema adhesivo de 1 paso Panavia ${ }^{\mathrm{TM}} \mathrm{F}$ y autoadhesivo Clearfil SA. Concluyeron que el uso del sellador que contiene eugenol reduce significativamente la resistencia adhesiva del poste de fibra, pero el grupo Panavia ${ }^{\mathrm{TM}} \mathrm{F}$ fue menos susceptible al efecto inhibidor de eugenol que los otros grupos evaluados. $^{20}$

Considerando lo anterior, el objetivo de este trabajo fue evaluar la influencia in-vitro de dos selladores endodónticos con diferentes composiciones sobre la resistencia adhesiva de los postes a través de los ensayos de resistencia adhesiva al desalojo (push-out), así mismo la eficacia del sellado a través del análisis de microfiltración y densidad radiográfica. La hipótesis nula a ser evaluada es que los diferentes selladores endodónticos tendrán un desempeño in-vitro similar.

\section{Materiales y Métodos}

Se utilizaron 55 dientes bovinos anteroinferiores extraídos de dimensiones similares y libres de tejidos periodontales, con ápice cerrado, seccionados al nivel de la unión amelocementaría, estandarizados a $16 \mathrm{~mm}$ de dimensión apicocervica. Las raíces fueron divididas aleatoriamente en tres grupos, dependiendo del cemento sellador a ser utilizado: control, VIARDEN® o ADSEAL ${ }^{\mathrm{TM}}$. Todas las raíces fueron obturadas utilizando puntas de gutapercha (SybroEndo, XF-M) asociadas al sellador de conductos correspondiente, el cual fue aplicado siguiendo las instrucciones del fabricante. Posterior a la obturación, postes de fibra de vidrio (Snowpost, \#40) fueron cementados en cada uno de los conductos utilizando el sistema Luting Cement DC (Prime-Dent®) asociado al sistema adhesivo Ligth Cure Denting/Enamel Bond (Prime-Dent $\left.{ }^{\circledR}\right)$.

Para la prueba se push out se seccionó cada muestra, el primer corte coronal de $1 \mathrm{~mm}$ se descartó debido a la sobreobturación del cemento. Se obtuvieron tres cortes por espécimen de $2 \pm 0,3 \mathrm{~mm}$ de grosor y se colocaron en agua desionizada $37^{\circ} \mathrm{C}$ por 7 días hasta que se realizó la prueba. Para la prueba mecánica se aplicó una fuerza en sentido ápico-coronal utilizando una máquina de ensayos universal (Instron Mod. 4465) a una velocidad de $1 \mathrm{~mm} / \mathrm{min}$, induciendo la carga sobre el cilindro metálico hasta expulsar el poste. Para calcular la 
resistencia adhesiva al desalojo $(\sigma)$, se utilizó la siguiente formula:

$$
\sigma=\mathrm{F} / \mathrm{A}
$$

Donde: $\mathrm{F}$ = la carga de ruptura; y $\mathrm{A}$ = área de superficie del endoposte $\left(\mathrm{mm}^{2}\right)$.

Para la prueba de microfiltración $(n=10)$, se realizó el tratamiento de endodoncia con la técnica anteriormente descrita. Una vez realizada la obturación, los dientes fueron almacenados $37^{\circ} \mathrm{C}$ por 7 días y, posteriormente se colocó una capa de barniz de uñas transparente en la parte externa de la raíz sin tocar el ápice del diente ni la abertura del conducto. Las raíces fueron luego sumergidas en una solución de azul de metileno al $2 \%$ durante siete días. Transcurrido ese tiempo, las raíces fueron montadas en tubos PVC con resina cristal y seccionadas en su eje longitudinal. Las muestras fueron observadas con un microscopio estereoscópico (Mobiloskop, Renfert) y la cantidad microfiltración de azul de metileno en sentido ápico-coronal fue medida en $\mathrm{mm}$. Para la prueba de densidad radiográfica, fueron tomadas y digitalizadas radiografías ortorradiales de las raíces de los dientes obturadas con los diferentes selladores $(n=10)$. Las imágenes digitalizadas fueron analizadas con el programa Image Tool for Windows, versión 2.00. Para el análisis, una porción del ápice del diente fue seleccionada y la densidad promedio en pixeles fue registrada.

Las pruebas estadísticas fueron realizadas utilizando el programa Sigma Plot 12.0. Los datos fueron analizados para verificar su distribución normal y la homogeneidad en la varianza. Los datos de la resistencia adhesiva fueron analizados a través de la prueba de KruskalWallis, mientras que los datos de microfiltración y densidad radiográfica fueron analizados con la prueba de ANOVA de una vía. Para todos los casos, un análisis complementario de Tukey fue realizado para detectar diferencias estadísticamente significativas entre los grupos. Para todas las pruebas estadísticas, se utilizó un nivel de significancia de $\alpha=0.05$.

\section{Resultados}

La Tabla 1 muestra la mediana y el rango intercuartílico de la resistencia adhesiva mediante la prueba de desalojo de los postes de fibra de vidrio cementados en los diferentes grupos. Los resultados, analizados a través de la prueba de Kruskal-Wallis, muestran que el grupo obturado con el sellador ADSEAL ${ }^{\mathrm{TM}}$ obtuvo valores significativamente mayores a los de los grupos obturados con el cemento Viarden $\AA(p<0.05)$, y mayores al grupo control $(p<0.05)$.
Tabla 1. Resistencia adhesiva al desalojo (MPa) de los postes de fibra de vidrio cementados en los diferentes grupos [Mediana (rango intercuartílico)]

\begin{tabular}{ll}
\hline Grupo & $\begin{array}{l}\text { Resistencia adhesiva } \\
(\mathrm{MPa})\end{array}$ \\
\hline ADSEAL $^{\mathrm{MM}}$ & $3.85(3.63-3.93)^{\mathrm{a}}$ \\
Viarden $^{\mathrm{B}}$ & $0.97(0.85-1.15)^{\mathrm{b}}$ \\
Control $^{\mathrm{b}}$ & $0.22(0.18-0.28)^{\mathrm{c}}$ \\
\hline
\end{tabular}

Letras superíndice ${ }^{(a-c)}$ diferentes indican diferencias estadísticamente significativas $(p<0.05)$

Las medias y desviaciones estándar de la microfiltración de azul de metileno están mostradas en la Figura 2. De acuerdo con el análisis estadístico, el grupo control mostró valores de filtración significativamente mayores que los grupos $A D S E A L^{T M}$ y Viarden ${ }^{\circledR}(p>0.05)$. De igual forma, las diferencias encontradas entre los selladores ADSEAL $^{\mathrm{TM}}$ y Viarden ${ }^{\circledR}$ fueron estadísticamente significativas.

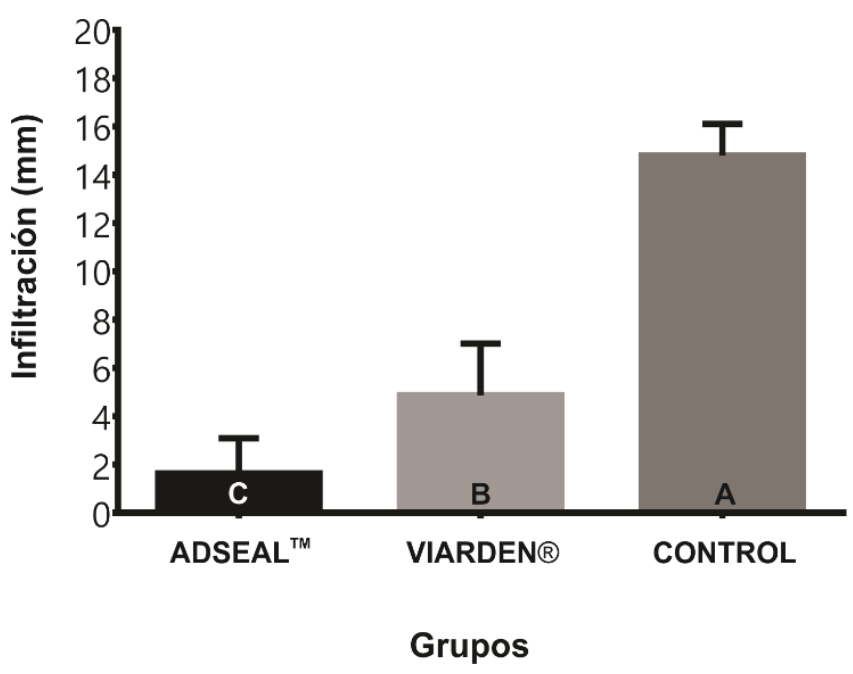

Figura 1. Media y desviación estándar de la microfiltración de azul de metileno. Diferentes letras en cada barra indican diferencias estadísticamente significativas $(p<0.05)$.

La Tabla 2 muestra los valores de densidad radiográfica de la región apical obtenidos. Los resultados del análisis demostraron que, con respecto al control, el uso de los selladores endodónticos ADSEAL $^{\mathrm{TM}}$ y Viarden $\AA$ aumentó significativamente el promedio de pixeles observados. 
Tabla 2. Pixeles de densidad radiográfica de los diferentes grupos [Promedio (DE)]

\begin{tabular}{ll}
\hline Grupo & $\begin{array}{l}\text { Densidad radiográfica } \\
\text { (pixeles) }\end{array}$ \\
\hline ADSEAL & $219.6( \pm 12.2)^{\mathrm{a}}$ \\
Viarden $^{\mathrm{a}}$ & $226.4( \pm 12.3)^{\mathrm{a}}$ \\
Control $^{\mathrm{a}}$ & $183.2( \pm 43.9)^{\mathrm{b}}$ \\
\hline
\end{tabular}

Letras superíndice $^{(a-b)}$ diferentes indican diferencias estadísticamente significativas $(p<0.05)$

\section{Discusión}

En este estudio, la efectividad in-vitro de dos selladores endodónticos de diferente composición fue evaluada a través de diversas técnicas de caracterización. De acuerdo con los resultados obtenidos, la resistencia adhesiva al desalojo y la microfiltración fueron materiales dependientes. Por otro lado, la densidad radiográfica no fue significativamente alterada por el uso de los diferentes selladores endodónticos utilizados. Considerando estos resultados, la hipótesis nula fue parcialmente rechazada.

La prueba de resistencia adhesiva al desalojo demostró que el sellador VIARDEN® obtuvo los valores más bajos $(p<0.05)$. El eugenol presente en los cementos a base de $Z O E$, es un molécula que se difunde fácilmente a través de los túbulos dentinarios, por lo que su presencia en tercios medios y cervicales de la raíz, es esperada. ${ }^{21} \mathrm{La}$ asociación entre la presencia de eugenol y la inhibición de la polimerización de materiales resinosos es un fenómeno ampliamente reportado en la literatura, ${ }^{22}$ y esta inhibición resulta en una deficiencia en la calidad del polímero resultante, afectando negativamente la capacidad adhesiva del material. ${ }^{3-5}$

Por otro lado, el grupo obturado con el sellador ADSEAL ${ }^{\mathrm{TM}}$ obtuvo los valores de resistencia adhesiva más elevados. De acuerdo con la composición proporcionada por el fabricante, $A D S E A L^{\mathrm{TM}}$ es un sellador endodóntico a base de resina, no habiendo componentes identificados con capacidad potencial para inhibir la polimerización de los materiales empleados para la adhesión del poste de fibra de vidrio. ${ }^{26}$ De igual forma, es posible que exista una unión química entre los componentes resinosos del sellador y los monómeros presentes en el sistema adhesivo y cemento dual utilizado para la cementación del poste de fibra de vidrio, lo que podría favorecer la resistencia adhesiva de estas restauraciones intrarradiculares. ${ }^{27}$

La evaluación de la microfiltración demostró que el grupo control, donde no se utilizó un sellador endodóntico, mostró el mayor promedio de filtración de azul de metileno $(p<0.05)$. Este resultado refuerza la idea de que el empleo de un sellador endodóntico se hace necesario para favorecer el sellado del material de obturación radicular. Se ha reportado en estudios previos que, la gutapercha, al no adherirse a las paredes de la dentina, no es capaz de proveer un sellado satisfactorio ${ }^{28}$, hecho que también pudo comprobarse en nuestro estudio.

Cuando se compararon los cementos $\operatorname{ADSEAL}^{\mathrm{TM}}$ y Viarden $\AA$, los resultados demostraron que el cemento a base de ZOE tuvo valores significativamente mayores de filtración $(p<0.05)$. Se ha reportado que los cementos a base de óxido de zinc y eugenol muestran una solubilidad elevada. Este alto grado de solubilidad es probablemente el resultado de la liberación del eugenol en exceso que no reaccionó, así como de la reacción de hidrólisis del eugenolato de zinc ya endurecido. ${ }^{29}$ Esta pérdida de material puede afectar negativamente la capacidad selladora de estos materiales. ${ }^{30}$ Por otro lado, los cementos a base de resina epóxica han demostrado tener una buena efectividad en cuanto sellado apical se refiere. Gracias a su composición química, el polímero resultante en este tipo de materiales posee un elevado entrecruzamiento, lo que lo hace ser un material con alta resistencia a la hidrólisis, disminuyendo así su solubilidad. $^{31}$ De hecho, gracias a que prácticamente pueden considerarse como insolubles, los selladores de conductos radiculares a base de resina pueden ser ideales para usarse como selladores endodónticos.

Finalmente, la densidad radiográfica de los selladores endodónticos fue determinada para conocer la radiopacidad de los mismos. La densidad radiográfica observada en estos materiales fue estadísticamente similar $(p>0.05)$, lo que sugiere que ambos materiales contienen un porcentaje semejante de agentes radiopacos dentro de su composición. ${ }^{32}$ El estudio de la radiopacidad de estos materiales es de suma importancia por tratarse de una propiedad física que permite ver el material de obturación endodóntico para verificar la calidad de la obturación. ${ }^{33}$

A realizarse la evaluación en el grupo control, sin sellador, el valor obtenido mediante el histograma es significativamente menor cuando se compara con los grupos obturados con $A D S E A L^{\mathrm{TM}}$ y Viarden®. La ausencia de material sellador evita la obturación adecuada del conducto, observándose zonas radiolúcidas a lo largo del espacio radicular entre los conos de gutapercha y las paredes del conducto, así como el ápice radiográfico, demostrando una vez más la incapacidad de la gutapercha para proveer, por sí sola, un sellado adecuado. ${ }^{34}$

\section{Conclusiones}

Los resultados de este estudio in-vitro sugieren que el uso de selladores endodónticos es indispensable para favorecer el desempeño del material utilizado para la 
obturación endodóntica. Así mismo, en este estudio fue posible demostrar que la composición química del material utilizado como sellador endodóntico tiene una influencia significativa en la filtración apical y en la resistencia adhesiva al desalojo de postes de fibra de vidrio cementados con resina dual. De entre los materiales utilizados, el sellador a base de resina epóxica parece tener el mejor desempeño, y puede considerarse como ideal para procedimientos endodónticos.

\section{Referencias}

1. Organization WH. Oral health surveys: basic methods. World Health Organization; 2013.

2. Kidd EAM, Fejerskov O. What constitutes dental caries? Histopathology of carious enamel and dentin related to the action of cariogenic biofilms. J Dent Res. 2004;83:35-8.

3. Ferreira-Nóbilo $\mathrm{N}$ de $\mathrm{P}$, de Sousa $\mathrm{M}$ da LR, Cury JA. Conceptualization of dental caries by undergraduate dental students from the first to the last year. Braz Dent J. 2014;25:529.

4. Mooney JB, Barrancos PJ. Operatoria Dental/ Dental Operation: Integracion Clinica/ Clinical Integration [Internet]. 4a. Médica Panamericana, editor. Buenos Aires: Médica Panamericana; 2006. Available from: https://books.google.com.mx/books?id=zDFxeYR8QWwC

5. Hargreaves KM, Berman LH, Cohen S. Obturación del sistema de conductos radiculares limpios y conformados. In: Mosby E, editor. Vías de la Pulpa [Internet]. 10th ed. Barcelona: Elsevier Health Sciences Spain; 2011. p. 349-88. Available from: https://books.google.com.mx/books?id=auG-aCkeoUgC

6. Kaur A, Shah N, Logani A, Mishra N. Biotoxicity of commonly used root canal sealers: A meta-analysis. J Conserv Dent JCD. 2015;18:83.

7. Song Y-S, Choi Y, Lim M-J, Yu M-K, Hong C-U, Lee K-W, et al. In vitro evaluation of a newly produced resin-based endodontic sealer. Restor Dent Endod. 2016;41:189-95.

8. Skupien JA, Sarkis-Onofre R, Cenci MS, MORAES RR de, Pereira-Cenci T. A systematic review of factors associated with the retention of glass fiber posts. Braz Oral Res. 2015;29:1-8.

9. Altmann ASP, Leitune VCB, Collares FM. Influence of eugenol-based sealers on push-out bond strength of fiber post luted with resin cement: systematic review and meta-analysis. J Endod. 2015;41:1418-23.

10. Mosharraf R, Zare S. Effect of the type of endodontic sealer on the bond strength between fiber post and root wall dentin. J Dent (Tehran). 2014;11:455.

11. Demiryürek EÖ, Külünk S, Yüksel G, Saraç D, Bulucu B. Effects of three canal sealers on bond strength of a fiber post. J Endod. 2010;36:497-501.

12. Menezes MS, Queiroz EC, Campos RE, Martins LRM, Soares CJ. Influence of endodontic sealer cement on fibreglass post bond strength to root dentine. Int Endod J. 2008;41:476-84.

13. Souza-Junior EJ, Bueno VCPS, Dias CTS, Paulillo LAMS Effect of endodontic sealer and resin luting strategies on pull-out bond strength of glass fiber posts to dentin. Acta Odontológica Latinoam. 2010;23:216-21.

14. Rached- Junior FJA, Sousa- Neto MD de, Souza- Gabriel AE, Duarte MAH, Silva- Sousa YTC. Impact of remaining zinc oxide- eugenol-based sealer on the bond strength of a resinous sealer to dentine after root canal retreatment. Int Endod J. 2014;47:463-9.

15. Alfredo E, Souza ES de, Marchesan MA, Paulino SM, GaribaSilva R, Sousa-Neto MD. Effect of eugenol-based endodontic cement on the adhesion of intraradicular posts. Braz Dent J. $2006 ; 17: 130-3$.

16. Izadi A, Azarsina M, Kasraei S. Effect of eugenol-containing sealer and post diameter on the retention of fiber reinforced composite posts. J Conserv Dent JCD. 2013;16:61.

17. Aleisa K, Alghabban R, Alwazzan K, Morgano SM. Effect of three endodontic sealers on the bond strength of prefabricated fiber posts luted with three resin cements. J Prosthet Dent. 2012;107:322-6.

18. Santos VJS, Veloso HHP, Sampaio FC, Araujo TP, Silva RB. Eugenol influence on the bond strength of intracanal

metallic cast posts bonded with resinous cement. Dental Press Endod. 2012;2:26-31.

19. Mosharraf R, Haerian A. Push-out bond strength of a fiber post system with two resin cements. Dent Res J (Isfahan). 2011;8:S88.

20. Özcan E, Cetin AR, Capar ID, Tunçdemir AR, Aydinbelge HA. Influence of eugenol on the push-out bond strengths of fiber posts cemented with different types of resin luting agents. Odontology. 2013;101:204-9.

21. Teixeira CDS, Pasternak B, Borges AH, Paulino SM, SousaNeto MD. Influence of endodontic sealers on the bond strength of carbon fiber posts. J Biomed Mater Res - Part B Appl Biomater. 2008;84:430-5.

22. Fujisawa S, Kadoma Y. Action of eugenol as a retarder against polymerization of methyl methacrylate by benzoyl peroxide. Biomaterials. 1997;18:701-3.

23. Farina AP, Chiela H, Carlini-Junior B, Mesquita MF, Miyagaki DC, Randi Ferraz CC, et al. Influence of Cement Type and Relining Procedure on Push-Out Bond Strength of Fiber Posts after Cyclic Loading. J Prosthodont. 2016;25:54-60.

24. Cecchin D, Farina AP, Souza MA, Da Cunha Pereira C. Effect of root-canal sealer on the bond strength of fiberglass post to root dentin. Acta Odontol Scand. 2011;69:95-100.

25. Cecchin D, Farina AP, Souza MA, Carlini-Júnior B, Ferraz CCR. Effect of root canal sealers on bond strength of fibreglass posts cemented with self-adhesive resin cements. Int Endod J. 2011;44:314-20.

26. Bohrer TC, Fontana PE, Wandscher VF, Morari VHC, Dos Santos SS, Valandro LF, et al. Endodontic Sealers Affect the Bond Strength of Fiber Posts and the Degree of Conversion of Two Resin Cements. J Adhes Dent. 2018;20:165-72.

27. Vilas-Boas DA, Grazziotin-Soares R, Ardenghi DM, Bauer J, de Souza PO, de Miranda Candeiro GT, et al. Effect of different endodontic sealers and time of cementation on push-out bond strength of fiber posts. Clin Oral Investig. 2018;22:1403-9.

28. Savariz A, González-Rodríguez MP, Ferrer-Luque CM. Longterm sealing ability of GuttaFlow versus Ah Plus using different obturation techniques. Med Oral Patol Oral Cir Bucal. 2010;15:936-41.

29. Schäfer E, Zandbiglari T. Solubility of root-canal sealers in water and artificial saliva. Int Endod J. 2003;33:660-9.

30. Ersahan S, Aydin C. Solubility and apical sealing characteristics of a new calcium silicate-based root canal sealer in comparison to calcium hydroxide-, methacrylate resin- and epoxy resinbased sealers. Acta Odontol Scand. 2013;71:857-62.

31. Case SL, O'Brien EP, Ward TC. Cure profiles, crosslink density, residual stresses, and adhesion in a model epoxy. 
Publicación semestral, Educación y Salud Boletín Científico Instituto de Ciencias de la Salud Universidad Autónoma del Estado de

Hidalgo, Vol. 8, No. 15 (2019) 151-157

Polymer (Guildf). 2005;46:10931-10840.

32.

Candeiro GTDM, Correia FC, Duarte MAH, Ribeiro-Siqueira DC, Gavini G. Evaluation of radiopacity, $\mathrm{pH}$, release of calcium ions, and flow of a bioceramic root canal sealer. J Endod. 2012;38:842-5.

33. Imai Y, Komabayashi T. Properties of a new injectable type of root canal filling resin with adhesiveness to dentin. J Endod. 2003;29:20-3.

34. Gambarini G, Tagger M. Sealing ability of a new hydroxyapatite-containing endodontic sealer using lateral condensation and thermatic compaction of gutta-percha, in vitro. J Endod. 1996;22:165-7. 